\title{
Influence of Medication Error among Medical and Non-medical Students in a Malaysian University
}

\author{
Lim Jing Hang ${ }^{1,2}$, Vetriselvan Subramaniyan ${ }^{1,2, *}$, Kok Xiaojun ${ }^{2}$, Mohamad Hazeeq bin Abu Bakar², Teoh Zheng Wei ${ }^{2}$, Meftah Jebriel \\ Meftah Alshtaiwi' ${ }^{2}$, Low Wei Ling ${ }^{2}$, Kanesh Ambihabathy ${ }^{2}$, Meram Azzani ${ }^{3}$ \\ 'Department of Pharmacology, Faculty of Medicine, MAHSA University, Bandar Saujana Putra, MALAYSIA. \\ 2Faculty of Medicine, MAHSA University, Bandar Saujana Putra, MALAYSIA. \\ ${ }^{3}$ Department of Community Medicine, Faculty of Medicine, MAHSA University, Bandar Saujana Putra, MALAYSIA.
}

\begin{abstract}
Background: Worldwide medication error is a common problem and inappropriate use of medicinal drugs is a major issue of drug resistance. Self-medication is a main contributor to human pathogen resistance to certain antibiotics. This research aims to investigate the prevalence, perception and predictors of self-medication among students in a Malaysian University. Methods: A total number of 317 students were included in this study. This cross-sectional study was conducted from Feb-May 2018. A convenience sample was taken from medical and non-medical university students. The association between the independents factors and the prevalence of self-medication was analysed using binary logistic regression. Data was analysed using SPSS version 23. Results: Among the respondents, $39.1 \%$ of them had recently practiced self-medication. The most common reason for self-medication was due to convenience $(50 \%)$ and the most common source of the medication is directly from pharmacies (46.4\%). Commonly used medicines were analgesics (44.4\%), cough and cold remedies $(31.0 \%)$ and antibiotics (9.9\%). The majority of students $(46.0 \%)$ experienced sleepiness after self-medication. Overall, the participants scored a mean 8 out of 10 questions for knowledge assessments
\end{abstract}

on self-medication. In the assessment of the participants' attitude towards self-medications, the participants agreed with 4 of 5 statements that are against self-medication. The probability of self-medication was higher among medical students compare to non medical students $(O R=2.039$, $P=0.025)$. In addition, students who are suffering from long term illness are at more risk of self medication $(O R=5.190, P=0.004)$. Conclusion: Selfmedication poses significant risk of toxicity upon misuse and proper selfmedication should be taught to all people through educational programs and better drug dispensing systems.

Key words: Self-medication, Over the counter, Medical and non-medical.

Correspondence

Dr. Vetriselvan Subramaniyan,

Associate Professor, Department of Pharmacology, Faculty of Medicine, MAHSA University, SP 2, Bandar Saujana Putra-42610, Jenjarom, Selangor, MALAYSIA.

Phone: +60172852512

Email: vetricology@gmail.com

DOI: 10.5530/jyp.2019.11.82

\section{INTRODUCTION}

Self-medication is a convenient yet inappropriate practice which is applied widely throughout the world. ${ }^{1}$ Every day millions of people practice self-medication with the use of medicine not specifically prescribed by a healthcare professional such as Over the Counter (OTC) medicines, leftover drugs and medicine not specifically prescribed for them. ${ }^{2}$ Selfmedication can be understood simply as "the use of medications without prior medical consultation regarding indication, dosage and duration of treatment".3Along with the ever-progressing industrial modernization of the world, pharmaceutical industries compete in the efficacy of both their drugs in curing illnesses and in their production to greatly outstand their opponents and obtain a greater profit. People nowadays no longer need to relive the hassle and fear they face during the "no prescription no medicine" days where doctors serve as the gatekeepers against their need for their medicine. The presence of physicians greatly ensures rational use of drugs and medicine which guards against risks of misuse such as resource wastage, drug abuse, drug toxicities and more importantly, antibiotic resistance. However, considering the advantages of self-medication in various aspects such as convenience, time consumption, cost and possibly avoiding the need to consult a professional "white coat" on what seemed to be feeble matters, people overlooked these factors and chose to practice self-medication over standard consultation. ${ }^{4}$ The drive towards self-medication is further endorsed by advice from relatives and close friends, media propaganda or even the pharmacists themselves, probing them further to skip over the standard diagnosis before proceeding to disease treatment. Certain governments even encourage selfmedication as a method to reduce doctors' consultation time along with its advantages stated above. ${ }^{5}$ While it is possible that self-medication can provide relief or even treat minor illnesses, the risks of misuse and its consequences greatly overshadow its benefits.

In Malaysia, self-medication is a common practice where pharmacies and convenience stores are permitted to sell certain types of medicine commercially without valid prescriptions from doctors, as is a commonly seen phenomenon in various other developing countries as well. ${ }^{6}$ People at home tend to stash away previously unfinished "leftover" medicine for future use, without knowledge of its shelf life and potential toxicities. This concept of frugality, without the guidance of proper education, exposes a person to serious risks that may bring harm towards his or her health, including various factors such as drug interactions, adverse effects, contraindications, dosage problems and so on.

The preclinical medical students are trained in the study of medically related subjects such as physiology, pathology and pharmacology. ${ }^{7}$ Their syllabus involved studying the mechanisms and pharmacokinetics of various drugs and medications used in different conditions and have studied the properties of each drug, their indications as well as their potential adverse reactions, contraindications and drug interactions. This exposes them to knowledge critical for the appropriate practice, if not 
Hang, et al.: Influence of Medication Error among Medical and Non-medical Students

termination, of self-medication. This exposure also enables the development and formation of their unique opinion towards such practices, which will prove as valuable information to the adjustment of this currently underdeveloped sector of modern medicine. In the light of such circumstances, the present study aims to investigate the prevalence, perception and predictors of self-medication among students in a Malaysian University. ${ }^{8}$ Globally, inappropriate use of antibiotics exhibited resistance to many antibacterial drugs such as vancomycin, methicillin etc. The development of resistance appalling high into certain antibiotics including clarithromycin, amoxicillin and metronidazole. ${ }^{9,10}$ Some developed and developing countries restricted to dispense antibiotics without proper drug prescription. On the contradictory, developed countries still explore high rate of medication error and this may influence by lack of professional education. Moreover, several studies stated that awareness of self-medication more important to university and school students. ${ }^{11,12}$

\section{METHODOLOGY}

A questionnaire-based cross-sectional, the survey was conducted from February 2018 through May 2018. The participants for this study are students of MAHSA University, Saujana Putra campus, located in Selangor, Malaysia. The students are separated into preclinical medical students [(Medical students Year 1(MY1) and Medical students Year 2 (MY2)] and non-medical students (NM) which include disciplines from engineering, business, accounting, human resource management and oil and gas management. All preclinical medical students as well as students from non-medically related faculties, aged between 18 and 25 are allowed to participate in the study. There are no exclusion criteria for this study. The data collected are included the gender, age of participants, course of participants, practice of participants on self-medication, knowledge of participants on self-medication and attitude of participants on self-medication. A self-administered questionnaire was developed. It was then pre-tested in on 20 students in order to assess the validity, reliability and relevance as a functional data collection tool.The questionnaire contained open and close ended questions. It consisted of 5 sections, each containing 5 to 10 questions that addresses the core issues of this study. For knowledge scores on self-medication, 1 point is awarded to the respondent for every "Yes" answered, amounting up to a maximum of 10 points. The knowledge score on self-medication is then categorized into two groups with the mean score taken as the cutoff point. The participants who scored same as or more than the mean score is labelled "Good", the participants who scored less than the mean is labelled "Poor". The attitude of participants towards self-medication consists of 4 categories, labelled as strongly disagree, disagree, agree and strongly agree. For attitude scores on self-medication, 1 point is awarded to the respondent for every "strongly disagree" answered, 2 points are awarded to the respondent for every "disagree" answered, 3 points are awarded to the respondent for every "agree" answered and 4 points are awarded to the respondent for every "strongly agree" answered, amounting up to a maximum of 10 points.

Descriptive statistics, including frequencies and percentages for categorical variables and mean and standard deviation for numerical variables, are calculated for each item in the questionnaire. The survey was developed after a comprehensive review of the related literature and consultation among the research team. It was face-validated via consultation with expert colleagues in the field and was also objectively validated for comprehensibility and clarity. "A written informed consent to participate in a study was obtained from all the participants and the principles of the Declaration of Helsinki were followed. Identifiable information was removed to ensure participant anonymity."
Ethical approval was obtained prior to the commencement of the study from the institutional ethics committee. The ethics committee approval number is $2018 / 1$ and protocol number is $2018 / 8 \mathrm{~B}$.

All the study variables with a $P$-value of $<0.05-0.3$ in the univariate logistic analysis were included in the final multivariate logit model. A logit model was developed to find out the probability of the self-medication among study participants. The results and relevant data are presented in tables. The result was interpreted using the $P$-value of $<0.05$, as a measure of the statistical significance. Data was analysed using SPSS version 23.

\section{RESULTS}

The study recorded a total of 317 valid responses from the participants, excluding incomplete responses. Among them, 39.1\% $(n=124)$ of them had recently practiced self-medication, specifically in the past 3 months. Here we explore the relationship between the participants' practice of self-medication against 3 variables, namely their course group, their gender and their age.

Table 1 shows the frequency of self-medication among the three different groups of participants which two of them are medical students which consist of year 1 and year 2 students while the other is non-medical students. The frequency of medical students of year 1 was $25.2 \%(n=80)$ out of 317 participants, where $36.3 \%(n=29)$ of them practiced self-medication in the past 3 months. While $32.5 \%(n=103)$ are year 2 medical students and $50.5 \%(n=52)$ them recently practiced self-medication. The remaining $42.3 \%(n=134)$ are non-medical students and $32.1 \%(n=43)$ of them recently practiced self-medication. Based on gender, the frequency of males was $39.7 \%$ ( $n=126)$ out of 317 participants, with $36.5 \%(n=46)$ of them having recently practiced self-medication. While the remaining $60.3 \%(n=191)$ are females and $40.8 \%(n=78)$ of them practiced selfmedication in the past 3 months (Table 1).

The mean age of participants is 20.88 years old $(\mathrm{SD}=1.57$, min. 18 , max. $25)$. The age of respondents is grouped into 2 groups, which are those who are 21 and below and those who are above 21 years old. The frequency of samples collected based on each age group. 65.9\% $(n=209)$ of participants are aged 21 and below, 38.3\% $(n=80)$ of whom practiced self-medication. The remaining $34.1 \%(n=108)$ of the respondents are aged above 21 years old, $40.7 \%(n=44)$ of which practiced self-medication in the past 3 months (Table 1 ).

Table 2 shows $7.5 \%(n=24)$ participants reported to have been suffering long term illnesses. A total of 26 complaints are recorded, with some participants responded to have one or more long term illnesses. The most common illness suffered among the respondents is Asthma, which amounted to a percentage of $34.6 \%(n=9)$. Other less common illnesses include Eczema 15.4\% ( $n=4)$, Gastritis 15.4\% ( $n=4)$, Sinusitis $11.5 \%$ $(n=3)$. The most common medicine that the students had consumed without prescription was analgesics by $44.4 \%(n=76)$, followed by cough and cold remedies by $31.0 \%(n=53)$, antibiotics by $9.9 \%(n=17)$ and others medicine amounting to a total of $14.6 \%(n=25)$ (Table 3$)$.

A major portion of the participants said that they had self-prescribed drug due to the factor of convenience, amounting to a total of $50 \%$ $(n=72)$ while the opinions of family members or friends, which is $29.9 \%$ $(n=43)$ was also an influential factor for self-medication practice by the students. Another $16.7 \%(n=24)$ of students had financial concerns against getting a doctor's consult. Problems with their doctors, $2.8(n=4)$ and other influencing factors, $0.7 \%(n=1)$ were the least influential factors for self-medication (Table 4).

Table 5 shows among the respondents, $68.5 \%(n=85)$ of respondents reported a self-perceived adverse drug reaction. The majority of students, experienced sleepiness $46.0 \%(n=64)$, rash, $4.3 \%(n=6)$ and abdominal 
Hang, et al:: Influence of Medication Error among Medical and Non-medical Students

discomfort, 3.6\% $(n=5)$. However, $18.0 \%(n=25)$ of respondents experienced other adverse effects. The majority of students, $46.4 \%(n=78)$ get their medication directly from pharmacies. Another 22.6\% $(n=38)$ of students get the medications from family or friends while $20.8 \%(n=35)$ of them considered previous prescriptions for their illnesses. However, $8.9 \%(n=15)$ of respondents consumed medicine which is prescribed for their family members or friends, while the remaining $1.2 \%(n=2)$ is sided by the students that had their medication from other sources (Table 6).

The mean score of self-medication knowledge is $8.02(\mathrm{SD}=2.263$, min. 0 , max. 10 ), with $67.5 \%$ participants scoring well in knowledge on selfmedication. While the mean score of attitude is 15.9 ( $\mathrm{SD}=3.587$, $\mathrm{min}$. 4 , max. 20), with $64.4 \%$ of participants having a good attitude on selfmedication. Table 7 shows that three factors are with $p$ value of $<0.05-0.3$ which they included in final logit model. Table 8 presents the results of the binary logistic regression analysis. the analysis showed that medical students are at higher risk of self meciation in compare to non medical students $(\mathrm{OR}=2.039, P=0.025)$. In addition, those suffering from long term illness are at more risk of self meicaltion $(\mathrm{OR}=5.190, P=0.004)$.

\section{DISCUSSION}

Our present study shows that $39.1 \%$ of the total 317 students have recently practiced self-medication. This result is in agreement with previous studies, ${ }^{13}$ where they found out that the prevalence of self-medication among healthcare students in Gondar University, Ethiopia is 38.5\%. In addition to that, the result is also consistent that the prevalence of self-medication of students in Mekelle University, Ethiopia is $43.24 \%$. The result, however, is also found to be comparatively lower than several previous studies. Previous studies reported that $55.2 \%$ prevalence in selfmedication among medical students in Egypt. In a study reflected 76\% of respondents from Karachi, Pakistan admitted to practice self-medication. ${ }^{14}$ Previous study stated that $79.9 \%$ of medical students in Belgrade, Serbia, reported to have previous experiences of self-medication. ${ }^{15}$ The prevalence of self-medication among premedical students in Pokhara, Nepal is $81.35 \% .{ }^{16}$ The prevalence of self-medication among students of University of Ljubljana, Slovenia is $92.3 \%$. The discrepancies in the prevalence of self-medication practice might be caused by the variance in drug use policies as well as perception towards self-medication among different countries and continents. ${ }^{17}$

In terms of the study, the majority of participants who practice self-medication belong to the medical course group, specifically from medical year $2(50.5 \%)$, compared to medical year $1(36.3 \%)$ and non-medical $(32.1 \%)$ course groups. This finding is in agreement with the study done by Lukovic et al. ${ }^{15}$ where they reported $58 \%$ of participants who selfmedicated are healthcare students. $40.8 \%$ females recently practiced selfmedication, which is more common than males (36.5\%). ${ }^{14}$ Our study also showed that there is no significant difference among different gender groups in their practice of self-medication $(p>0.05)$. This result is consistent with other study, where they found no significance between gender and self-medication practices. Also, previous studies found out that females practiced self-medication more than males. ${ }^{18}$ On the other hand, studies stated that there is a significant difference between gender and self-medication practice, where females tend to self-medicate more than males. ${ }^{19}$ With the supporting of another study also found an association between gender and drugs used for self-medication practice. ${ }^{16}$ The $40.7 \%$ participants aged above 21 have recently practiced self-medication, compared to $38.3 \%$ of participants who are 21 and below. Our study showed that there is no significant difference among different age groups in their practice of self-medication $(p>0.05)$. This result agrees with the previous research, where she concluded that there is no significant difference between age and self-medication practice. ${ }^{20}$ Moreover, previous studies found that there is a negative correlation between self-
Table 1: Frequency of self-medication among study participants.

\begin{tabular}{cccc}
\hline Variables & Total & \multicolumn{2}{c}{ Self-Medicated } \\
\cline { 3 - 4 } & & $\mathrm{N}$ & $\%$ \\
\hline Course group & & 29 & 36.3 \\
Medical Year 1 & 80 & 52 & 50.5 \\
Medical Year 2 & 103 & 43 & 32.1 \\
Non-Medical & 134 & & \\
Gender & & 46 & 36.5 \\
Male & 126 & 78 & 40.8 \\
Female & 191 & & \\
Age Group & & 80 & 38.3 \\
21 and below & 209 & 44 & 40.7 \\
Above 21 & 108 & & \\
\hline
\end{tabular}

Table 2: Long term illnesses among study participants.

\begin{tabular}{ccc}
\hline Long term Illnesses & N & $\%$ \\
\hline Gastro-esophageal Reflux Disease & 1 & 3.8 \\
Asthma & 9 & 34.6 \\
Sinusitis & 3 & 11.5 \\
Eczema & 4 & 15.4 \\
Gastritis & 4 & 15.4 \\
Hypotension & 1 & 3.8 \\
Migraine & 1 & 3.8 \\
Polycystic Ovarian syndrome & 1 & 3.8 \\
Scoliosis & 2 & 7.7 \\
Total & $\mathbf{2 6}$ & $\mathbf{1 0 0 . 0}$ \\
\hline
\end{tabular}

Table 3: Frequency of medicines used among study participants.

\begin{tabular}{ccc}
\hline Medicine Used & N & $\%$ \\
\hline Analgesics & 76 & $44.4 \%$ \\
Antibiotics & 17 & $9.9 \%$ \\
Cough and Cold & 53 & $31.0 \%$ \\
Other & 25 & $14.6 \%$ \\
Total & 171 & $100.0 \%$ \\
\hline
\end{tabular}

Table 4: Self-medication influencing factors.

\begin{tabular}{ccc}
\hline Influencing Factors & N & $\%$ \\
\hline Advice from Family/ & 43 & $29.9 \%$ \\
Friends & & \\
Problems with Doctor & 4 & $2.7 \%$ \\
Financial Cost & 24 & $16.7 \%$ \\
Convenience & 72 & $50 \%$ \\
Other & 1 & $0.7 \%$ \\
Total & $\mathbf{1 4 4}$ & $\mathbf{1 0 0 . 0 \%}$ \\
\hline
\end{tabular}

medication and respondents' age. Further investigations are needed to determine if age is a significant factor in the practice of self-medication. In regard to long-term illness, we found that 7.5\% $(n=24)$ out of 317 participants suffered from long term illness. Among them, Asthma (34.6\%) is the most common long-term illness suffered by the participants. In an- 
Hang, et al:: Influence of Medication Error among Medical and Non-medical Students

Table 5: Reported adverse effects of self-medication among study participants.

\begin{tabular}{ccc}
\hline Adverse Effects & N & $\%$ \\
\hline None Felt & 39 & $28.0 \%$ \\
Sleepiness & 64 & $46.0 \%$ \\
Abdominal Discomfort & 5 & $3.6 \%$ \\
Rash & 6 & $4.3 \%$ \\
Other & 25 & $18.0 \%$ \\
Total & $\mathbf{1 3 9}$ & $\mathbf{1 0 0 . 0 \%}$ \\
\hline
\end{tabular}

Table 6: Sources of obtaining medicine among study participants.

\begin{tabular}{ccc}
\hline Medicine Source & N & $\%$ \\
\hline Family/Friends & 38 & $22.6 \%$ \\
Past Prescriptions & 35 & $20.8 \%$ \\
Prescriptions of Family & 15 & $8.9 \%$ \\
Members & 78 & $46.4 \%$ \\
Requested from & & \\
Pharmacy & 2 & $1.2 \%$ \\
Other & $\mathbf{1 6 8}$ & $\mathbf{1 0 0 . 0 \%}$ \\
Total & & \\
\hline
\end{tabular}

Table 7: Univariate logistic regression analysis of factors associated with self-medication.

\begin{tabular}{|c|c|c|c|}
\hline \multirow[t]{2}{*}{ Variables } & \multicolumn{3}{|c|}{ Self-medicated } \\
\hline & OR & $\mathrm{Cl}$ & P-value \\
\hline \multicolumn{4}{|l|}{ Age group } \\
\hline$>21$ years & 0.877 & $0.515-1.495$ & 0.631 \\
\hline \multicolumn{4}{|c|}{ Course group } \\
\hline Medical & 1.9908 .02 & $1.175-3.372$ & $0.010^{*}$ \\
\hline \multicolumn{4}{|l|}{ Gender } \\
\hline Male & 0.871 & $0.514-1478$ & 0.609 \\
\hline \multicolumn{4}{|c|}{$\begin{array}{c}\text { Has long term } \\
\text { illness }\end{array}$} \\
\hline Yes & 4.750 & $1.563-14.437$ & $0.006^{*}$ \\
\hline \multicolumn{4}{|c|}{ Level of knowledge } \\
\hline Good & 1.667 & $0.952-2.918$ & 0.740 \\
\hline \multicolumn{4}{|c|}{ Level of attitude } \\
\hline Good & 1.532 & $0.892-2.630$ & 0.122 \\
\hline
\end{tabular}

*Significant at $P<0.05$

other study revealed, ${ }^{15} 2.7 \%(n=35)$ respondents claimed to be suffering from chronic somatic illnesses, such as mitral valve prolapses, Polycystic Ovarian Syndrome (PCOS), scoliosis and Diabetes.

Analgesics (44.4\%) is the most commonly consumed medicine among the participants and a majority of participants self-medicated using paracetamol (46.3\%). ${ }^{13}$ Previous study reflected $87.2 \%$ reported using analgesics, mostly for prophylactic purposes (59.9\%). Also found that $92 \%$ participants consumed paracetamol for their illnesses, sometimes along with other drugs such as antacids and antibiotics. ${ }^{21}$ The research report stated that antipyretics are the most common drugs consumed (33\%), followed by antibiotics (26.2\%) and analgesics (18.89\%). ${ }^{16}$ Previous studies stated paracetamol and NSAIDs are the most commonly used class
Table 8: Multivariate logistic regression analysis of factors associated with self-medication.

\begin{tabular}{cccc}
\hline Variables & \multicolumn{3}{c}{ Self-medicated } \\
\cline { 2 - 4 } & OR & $\mathrm{Cl}$ & P-value \\
\hline $\begin{array}{c}\text { Course group } \\
\text { Medical }\end{array}$ & 2.039 & $1.094-3.800$ & $0.025^{*}$ \\
Having long term illness \\
$\begin{array}{c}\text { Yes } \\
\text { Level of attitude } \\
\text { Good }\end{array}$
\end{tabular}

${ }^{\star}$ Significant at $P<0.05$

of drugs among their subjects. ${ }^{18}$ Previous study reported that analgesics are the most commonly self-prescribed drugs, followed by Vitamins and antipyretics and the analgesics $(88.3 \%)$ are the most common drugs consumed. Most participants claimed that they practiced self-medication due to the factor of convenience (50\%). This finding is in agreement with $53 \%$ respondents thought self-medication is more convenient compared to getting a professional consult with a doctor, followed by ease of access (51\%) and time saving (42\%). ${ }^{22}$ Moreover, the previous experience is the most common factor in influencing self-medication practice. ${ }^{14}$

A majority (68.5\%) of respondents reported to have experienced sideeffects from the consumed self-medication drug. The majority of which suffered sleepiness $(46.0 \%)$, followed by other symptoms $(18 \%)$, rash (4.3\%) and abdominal discomfort (3.6\%). Other adverse effects include rash and abdominal discomfort. From here we can infer that while there is a risk of suffering adverse effects from self-medication, the adverse effects are mostly mild. In regard to source of medicine, a majority of respondents claimed to have obtained their medications directly from pharmacies $(46.4 \%)$. The same result is obtained from other research and stated that $27 \%$ participants obtained it from pharmacies. ${ }^{13}$ Overall, the participants scored a mean 8 out of 10 questions for knowledge assessments on self-medication, with $67.5 \%$ participants scoring well in knowledge on self-medication. Also, $82 \%$ of the participants perceived their knowledge levels of common OTC medications were moderate to low. ${ }^{21}$

In regards to participants' attitude towards self-medication and compared the responses against different variables. Overall, $64.4 \%$ are against self-medication. This research finding is consistent with previous research, where $52.3 \%$ of their participants disagreed with its practice. ${ }^{18}$ This result also contradicts several previous researches. Previous study shows that $55.5 \%$ participants agree with the practice of self-medication. Also agrees that $77.5 \%$ of their participants agree with the practice of self-medication as part of self-care. ${ }^{21}$ This study shows that medical students tend to practice self-medication more than non-medical students. This result contradicts with the previous study, where they found no significant differences between course groups and self-medication practices. However, this finding is consistent, where they stated in their study that health care students practice significantly more self-medication than non-healthcare students. ${ }^{15}$ This might be due to increased understanding of medical students towards drugs and illnesses which increased their confidence in practicing self-medication. In addition, students who are suffering of long term illness are at higher probability of self-medication.

\section{CONCLUSION}

Our study concluded that self-medication is more common among both medical and non-medical students in MAHSA University, Malaysia. This study revealed a significant difference among course groups in their 
practice of self-medication, knowledge and attitude score of self-medication. Based on study findings, we can conclude that strict legal regulations regarding control of selling medications should be implemented. Education campaign regarding this issue should also be considered, because self-medication among young students can indicate a serious risk to professionalism especially in medicine.

\section{CONFLICT OF INTEREST}

The authors declare that they have no competing interests.

\section{ABBREVIATIONS}

OTC: Over the counter; MY1: Medical student's year 1; MY2: Medical student's year 2; SD: Standard deviation; PCOS: Polycystic Ovarian Syndrome; NSAIDs: Non steroidal anti-inflammatory drugs.

\section{REFERENCES}

1. Chun HL, Fong CC, Sheng DH, Hsueh YC, Li JH, Ming KY. Inappropriate selfmedication among adolescents and its association with lower medication literacy and substance use. PLoS One. 2017;12(12):1-14.

2. Ocan M, Obuku EA, Bwanga F, Akena D, Richard S, Ogwal-Okeng J, et al. Household antimicrobial self-medication: A systematic review and meta-analysis of the burden, risk factors and outcomes in developing countries. BMC Public Health. 2015;15:1-11.

3. Iqbal AM, Ghulam R, RabiaT, Marriam S, Siddiqullah, Shujauddin M. Prevalence and pattern of self-medication in Karachi: A community survey. Pak J Med Sci. 2015;31(5):1241-5.

4. Tanya B, Barton LM, Tatiana T, Dena RE, Ira WB. Challenges to physician-patient communication about medication use: A window into the skeptical patient's world. Patient Prefer Adherence. 2012;6:11-8.

5. Darshana B. Self-medication: A current challenge. J Basic Clin Pharm. 2014;5(1):19-23.

6. Rosalind M, Goodman C. Performance of retail pharmacies in low- and middle-income Asian settings: A systematic review. Health Policy and Planning. 2016;31(7):940-53

7. So JY, Jin SJ. Changes of academic performance by integration between basic and clinical medicine in pre-clerkship medical education. Korean J Med Educ. 2018;30(3):209-18.

8. Haranath PS. Medical curriculum and pharmacology: An appraisal. Indian J Pharmacol. 2016;48(Suppl 1):S10-3.
9. Gould IM. Treatment of bacteraemia: Meticillin-resistant Staphylococcus aureus (MRSA) to vancomycin-resistant S. aureus (VRSA) Int J Antimicrob Agents. 2013;42(Suppl):S17-21.

10. McKinnell JA, Miller LG, Eells SJ, Cui E, Huang SS. A systematic literature review and meta-analysis of factors associated with methicillin-resistant Staphylococcus aureus colonization at time of hospital or intensive care unit admission. Infect Control Hosp Epidemiol. 2013;34(10):1077-86.

11. Almalak H, Albluwi Al, Alkhelb DA, Alsaleh HM, Khan TM, Hassali MA, et al. Students' attitude toward use of over the counter medicines during exams in Saudi Arabia. Saudi Pharm J. 2014;22(2):107-12.

12. Donkor ES, Tetteh QPB, Nartey P, Agyeman IO. Self-medication practices with antibiotics among tertiary level students in Accra, Ghana: A cross-sectional study. Int J Environ Res Public Health. 2012;9(10):3519-29.

13. Abay SM, Amelo W. Assessment of Self-Medication Practices among Medical, Pharmacy and Health Science Students in Gondar University, Ethiopia. J Young Pharm. 2010;2(3):306-10.

14. Zafar SN, Syed R, Waqar S, Zubairi AJ, VaqarT, Shaikh M, et al. Self-medication amongst university students of Karachi: prevalence, knowledge and attitudes. J Pak Med Assoc. 2008;58(4):214-7.

15. Jasminka AL, Vladimir M, Tatjana P, Goran T, Nevena R, Danijela A. Self-Medication Practices and Risk Factors for Self-Medication among Medical Students in Belgrade. Serbia. 2014;9(12):1-14.

16. Indrajit B, Brijesh S, Rajesh KG, Annavarapu A, Bedanta R, Pugazhandhi B, et al. Self-medication practice among preclinical university students in a medical school from the city of Pokhara, Nepal. Nepal J Epidemiol. 2016;6(2):574-81.

17. Klemenc KZ, Hladnik Z, Kersnik J. Self-medication among healthcare and non-healthcare students at University of Ljubljana, Slovenia. Med Princ Pract. 2010;19(5):395-401.

18. Girma BG, Diriba AG, Zerihun AK, Derbew FB, Abera HB, Mussie GH, et al. SelfMedication Practices among Health Sciences Students: The Case of Mekelle University. Journal of Applied Pharmaceutical Science. 2011;01(10):183-9.

19. El ENF, Ez EHS. Knowledge, attitude and practice of medical students towards self-medication at Ain Shams University, Egypt. J Prev Med Hyg. 2011;52(4):196-200.

20. Asiyeh P, Firoozeh M. Self-medication among students in Isfahan University of Medical Sciences based on Health Belief Model. J Educ Health Promot. 2014;3:1-10

21. Alemseged B, Edomgenet G, Askalech D, Estifanos P, Kedija A, Minyahil A Knowledge, Attitude and Practice of Self Medication among Pharmacy Students of Rift Valley University, Abichu Campus, Addis Ababa, Ethiopia. J Health Med Inform. 2017;8(3):269.

22. Sandip BP. Self-medication: Awareness and Attitude among undergraduate medical students in a tertiary care medical college, Dhule. National Journal of Community Medicine. 2015;6(2):198-202.

Article History: Submission Date : 29-08-2019; Revised Date : 19-09-2019; Acceptance Date : 01-10-2019

Cite this article: Hang LJ, Subramaniyan V, Xiaojun K, Bakar MHA, Wei TZ, Alshtaiwi MJM, Ling LW, Ambihabathy K, Azzani M. Influence of Medication Error among Medical and Non-medical Students in a Malaysian University. J Young Pharm. 2019;11(4):399-403. 